\title{
The Effect of Salbutamol Nebul Treatment on Systolic Pulmonary Artery Pres- sure in Newly Diagnosed Patients with Chronic Obstructive Pulmoner Disease
}

\author{
Cosgun $\mathrm{A}^{* 1}$ and Akpinar $\mathrm{E}^{2}$ \\ ${ }^{1}$ Department of Cardiology, Cubuk State Hospital, Ankara, Turkey \\ ${ }^{2}$ Department of Pulmonology, Cubuk State Hospital, Ankara, Turkey
}

*Corresponding author: Cosgun A, Department of Cardiology, Cubuk State Hospital, Ankara, Turkey, Tel: +90 03128378110, E-mail: ayhancosgun@gmail.com

Citation: Cosgun A, Akpinar E (2018) The Effect of Salbutamol Nebul Treatment on Systolic Pulmonary Artery Pressure in Newly Diagnosed Patients with Chronic Obstructive Pulmoner Disease. J Respir Dis Treat 1(1): 102. doi: 10.15744/2767-4649.1.102

Received Date: February 04, 2018 Accepted Date: April 13, 2020 Published Date: April 15, 2020

\begin{abstract}
Summary
Backround: The occurrence of pulmonary hypertension [PHT] in patients with chronic obstructive pulmoner disease [COPD] usually indicates progression of the disease and poor prognosis. The aim of this study is to investigate the effect of oxygen and bronchodilator treatment on systolic pulmonary artery pressure induced by exertion test in patients with COPD.

Patients and Method: Thirty patients who had not previously received bronchodilator treatment and detected obstruction findings in the respiratory function test were included in the study. Resting systolic pulmonary arterial pressures [sPAP] were measured by transthoracic echocardiography. Patients were then enrolled in the effort test. Patients' sPAP were measured again within 60 seconds after the end of the effort test. The patients were treated with 1 ampoule salbutamol nebulization for 30 minutes. Resting sPAP of the patients were measured. Patients were then subjected to a second effort test and within 60 seconds the patient's sPAP were measured again.

Results: After effort test before and after salbutamol treatment, the sPAP values in the study group were $40.01+\%-5.94$ and $35.33 \pm$ $4.88 \mathrm{~mm} \mathrm{Hg}$, propectively.

Conclusion: There was no significant difference in resting sPAP values before and after treatment with salbutamol nebula for $30 \mathrm{~min}$ in the study group. However, there was a significant difference between the measured sPAP levels before and after the treatment. This suggests that the treatment is a positive effect of increased pressure with exercise.
\end{abstract}

Keywords: Chronic Obstuctive Lung Disease; Pulmonary Arterial Pressure; Exercise Testing; Salbutamol

\section{Backround}

COPD may not only be a functional disorder of the lungs, but may also have systemic effects [1]. Extrapulmonary findings are described in skeletal muscle, bone and cardiovascular system [2]. It is thought that low-intensity chronic inflammation may cause oxidative stress and endothelial dysfunction, thus leading to ischemic heart disease or stroke [3,4]. The occurrence of pulmonary HT in patients with COPD usually indicates progression of the disease and poor prognosis. Pulmonary arterial hypertension $[\mathrm{PAH}]$ is diagnosed when mean pulmonary arterial pressure exceeds $25 \mathrm{~mm}-\mathrm{Hg}$ at rest or $30 \mathrm{~mm}-\mathrm{Hg}$ during exercise. Right heart catheterization is the gold standard method for measuring pulmonary artery pressure. In addition, echocardiography can be used for this purpose [5]. PAH is present in approximately 50\% of patients with COPD, varying according to diagnostic methods [6-9]. The pulmonary artery pressure may increase significantly during exercise, which may lead to an erroneous assessment of exercise capacity. It has been determined that pulmonary artery pressure increases with age in healthy people. However, an increase in pressure in patients with COPD may result in a higher load in the heart and circulation, and reduced exercise tolerance [10-12]. The development of PHT in patients with COPD is an important influence that may transform the disease into pulmonary heart disease [13]. The pathogenesis of PHT in COPD has not yet been clearly elucidated, but it is thought that oxidative stress plays a role [14-18]. Although the negative effect of physical exercise on pulmonary artery pressure in patients with newly diagnosed and untreated COPD is known, its effect on the prognosis of the disease is still unclear [19-22]. The aim of this study is to investigate the effect bronchodilator treatment on systolic pulmonary artery pressure induced by exertion test in patients with COPD. 


\section{Patients}

Thirty patients admitted to the chest diseases polyclinic between April and December 2017, who had not previously received bronchodilator treatment and detected obstruction findings in the respiratory function test were included in the study. The median age of the study group, consisting of 16 female and 14 male patients, was calculated as $51.06 \pm 12.88$ (21-74). The control group included 33 patients who were referred to the cardiology polyclinic with chest pain, who had no COPD, had an indication for effort test, and were negative in the effort test. The median age of the control group, which consisted of 16 female and 17 male patients, was calculated as $46.66 \pm 8.33$ (31-68). In both groups, patients with a systemic disease such as liver, heart, kidney, systemic hypertension, diabetes mellitus were excluded from the study. There was no statistically significant difference between demographic information of two groups.

\section{Methods}

The study group was initially subjected to respiratory function test. The detection of forced expiratory volume in $1 \mathrm{~s}$ [FEV1] / forced vital capacity [FVC] $<70$ in these patients was accepted as obstruction finding [23]. Resting systolic pulmonary arterial pressures [sPAP] were measured by transthoracic echocardiography in 30 patients who had obstructive findings. Patients were then subjected to effort test using the standard Bruce protocol [24]. The test was terminated when $88 \%$ of the target heart rate was reached. The patients' sPAP were measured again within 60 seconds after the end of the effort test. After at least 4 hours of rest, the patients were treated with 1 ampoule salbutamol nebulization for 30 minutes. Resting sPAP were measured by transthoracic echocardiography of the patients in the study group. Patients were then subjected to a second effort test and within 60 seconds the patient's sPAP were measured again. Transthoracic echocardiography was performed on 33 patients in the control group and resting sPAP were measured. Patients were then enrolled in the effort test. The test was terminated when $88 \%$ of the target heart rate was reached. Patients' sPAP was measured again within 60 seconds after the end of the effort test.

\section{Statistical Analysis}

SPSS software [Version X; IBM, Armonk, NY, USA] 20.0 was used for the collection and analysis of the data. Measurements were expressed as mean values with \pm standard deviation. Analyzes were performed using t-test. For all tests $\mathrm{p}<0.05$, the differences are statistical significance.

\section{Results}

Resting sPAP was $28.93 \pm 4.18 \mathrm{~mm}-\mathrm{Hg}$ in the study group before the effort test, while this value was $18.88 \pm 2.50 \mathrm{~mm}-\mathrm{Hg}$ in the control group. The difference between the two values was statistically significant $[p=0.00001, t=12.72481]$. After the effort test, the sPAP in the study group was $40.01 \pm 5.94 \mathrm{~mm}-\mathrm{Hg}$, while the control group was $26.38 \pm 1.8 \mathrm{~mm}-\mathrm{Hg}$. The difference between the two values was statistically significant $[\mathrm{p}=0.00001 \mathrm{t}=15.75405]$. In the study group there was a statistically significant difference between the resting sPAP and the sPAP after the effort test $[\mathrm{p}=0.0001, \mathrm{t}=10.26476]$ (Figure 1 ). There was also statistically significant difference between the resting sPAP and and the sPAP after the effort test in the control group [ $\mathrm{p}<0.00001, \mathrm{t}=20.55237$ ] (Figure 2).

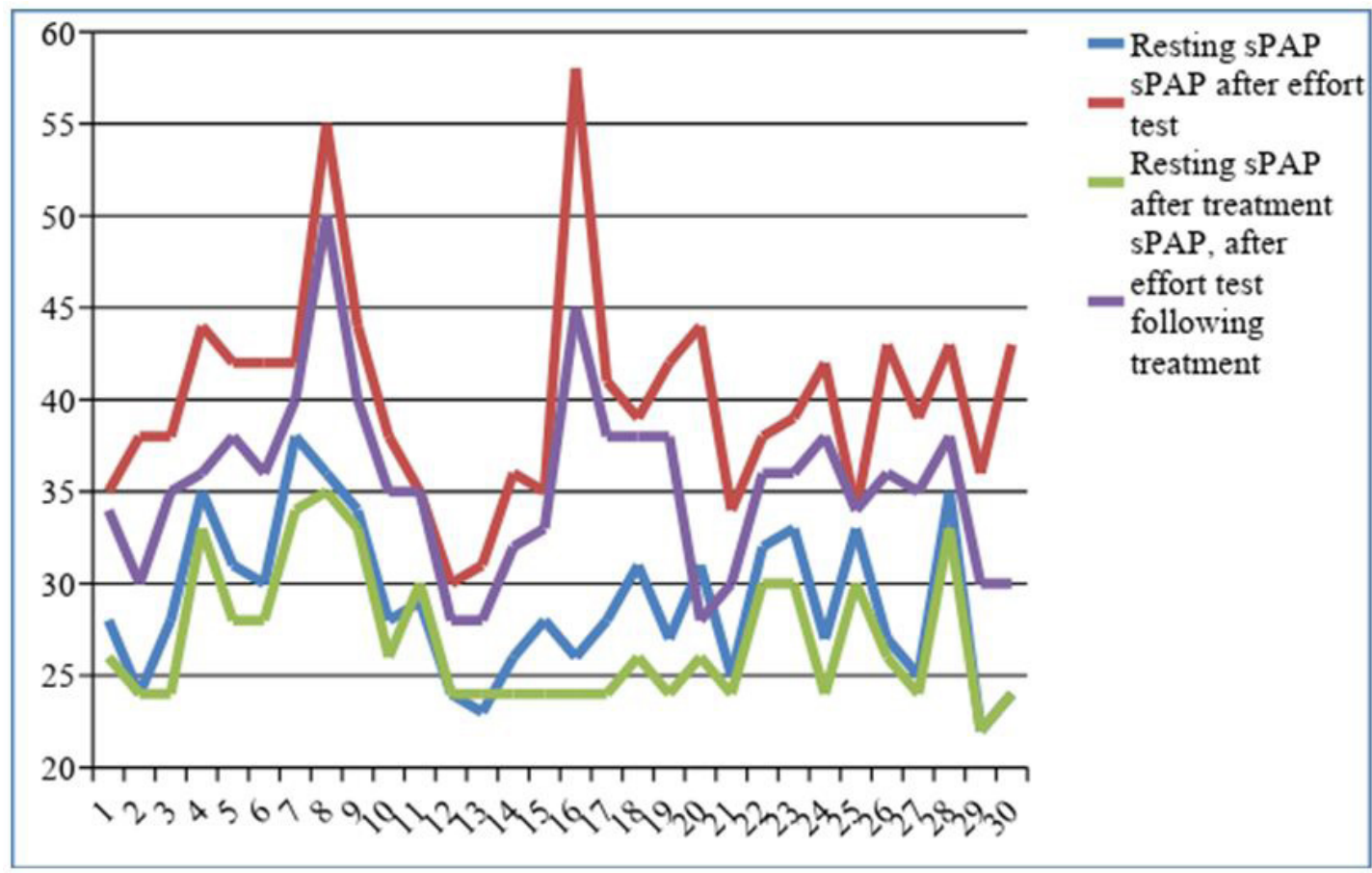

Figure 1: sPAP values of newly diagnosed COPD patients 


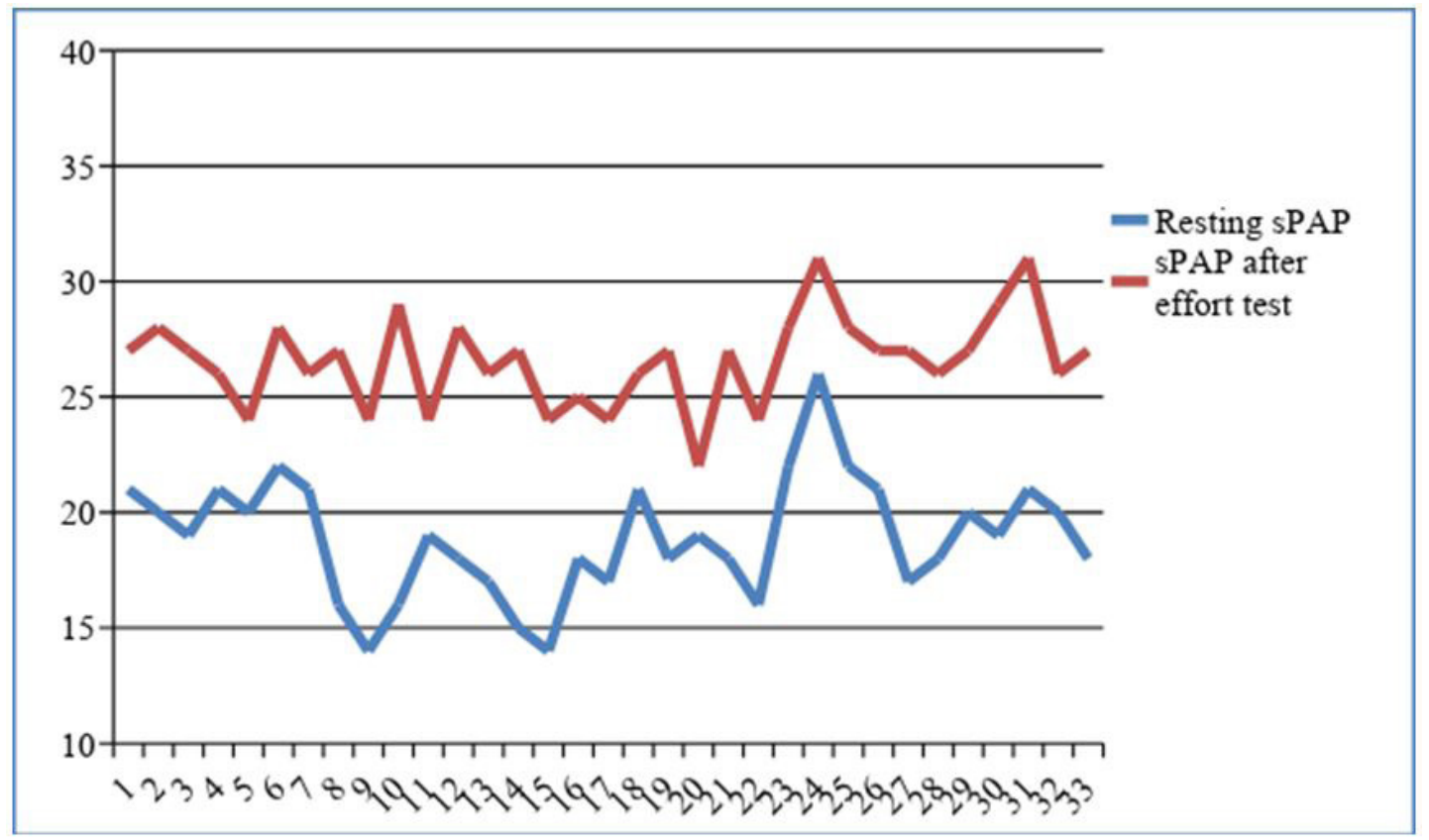

Figure 2: sPAP values of control group

Resting sPAP was $27.7 \pm 3.82 \mathrm{~mm}-\mathrm{Hg}$ after salbutamol nebul treatment applied for $30 \mathrm{~min}$ in the study group, and sPAP $35.33 \pm$ $4.88 \mathrm{~mm}-\mathrm{Hg}$ was measured after the effort test after treatment. The difference between the two values was statistically significant $[\mathrm{p}<0.001, \mathrm{t}=9.1913]$. In the study group, resting sPAP values before and after $\mathrm{O} 2+$ salbutamol treatment were $28.93 \pm 4.18$ and $27.7 \pm 3.82 \mathrm{~mm}-\mathrm{Hg}$, respectively. There was no statistically significant difference between the two values [ $\mathrm{p}=0.127451 \mathrm{t}=1.148913$ ] In the study group, the sPAP values before and after the salbutamol nebula treatment were $40.01 \pm 5.94 \mathrm{and} 35.33 \pm 4.88 \mathrm{~mm}-\mathrm{Hg}$, respectively. The difference between the two values was statistically significant $[\mathrm{p}=0.000034 \mathrm{t}=4.276995]$.

\section{Discussion}

A significant difference between the study group and the control group between resting pulmonary arterial pressures measured before and after the first effort test was shown to be appropriate for study and control group study purposes. In our prospective study, the fact that salbutamol nebula therapy is a statistically significant decrease in effort-induced sPAP in patients with first diagnosed COPD is an important result that should be evaluated in COPD patients. Secondary pulmonary hypertension results from sustained vasoconstriction and structural alterations to the pulmonary vascular bed. The major stimuli that are responsible for these changes are chronic alveolar hypoxia and chronic inflammation [25]. In spite of the effect of pulmonary blood flow which increases physiologically several times during exercise, the less increase of pulmonary artery pressure is explained by decrease in pulmonary vascular resistance. According to the results of our study, a greater increase in the sPAP values with effort in the study group than in the control group suggests that this decrease in adaptation may have occurred in COPD patients. The significant increase in sPAP before and after the effort test in both groups suggests that exercise contributes to the increase in pulmonary artery pressure. This is a finding that is physiologically accepted and expected [20]. However, the study group found that the difference between sPAP and sPAP after exercise was greater than in the control group suggests that patients with COPD are at risk for developing cardiac complications. The increased risk of cardiac complications has been shown in some previous studies [21,22,27-29]. Pulmonary artery pressure usually increases with age. Lam et al. in the study they performed, they determined that the pulmonary artery pressure measured by right heart catheterization was $14.0 \pm 3.3 \mathrm{~mm} \mathrm{Hg}$ in healthy subjects and that they increased in proportion to the age during exercise, especially up to $26 \mathrm{~mm}-\mathrm{Hg}$ above the age of 50 years [29]. Spiekerkoetter et al. have shown that salbutamol has beneficial acute effects on pulmonary function, blood gas and hemodynamics in patients with primary pulmonary hypertension [30]. However, there is limited research on the effect of beta 2 agonists on pulmonary artery pressure induced by effort in COPD patients. However, there are limitations of our work. The gold standard method of pulmonary artery pressure measurement is right heart catheterization. Another limitation is that the pressure was not measured during the effort test and was measured within 60 seconds of the end of the effort test. Echocardiography was performed after the effort test because it would be technically difficult to measure the sPAP during exercise and could affect the results of the test.

\section{Conclusion}

There was no significant difference in resting sPAP values before and after treatment with salbutamol nebula therapy for 30 min in the study group. However, there was a significant difference between the measured sPAP levels stimulated by exercise before and after the treatment. This suggests that the treatment is a positive effect of increased pressure with exercise. The fact that exercise-related systolic PHT is significantly reduced in COPD patients with treatment has been shown in this study, suggesting 
that treatment may also have a detrimental effect on cardiac complications. More extensive and ongoing studies are needed to determine the effect of treatment on mortality and morbidity of patients with COPD and PHT.

\section{Conflict of Interest}

The authors declare that they have no conflict of interest.

\section{Ethical Approval}

All procedures performed in studies involving human participants were in accordance with the ethical standards of the institutional and/or national research committee and with the 1964 Helsinki declaration and its later amendments or comparable ethical standards.

\section{References}

1. Barnes PJ, Celli BR (2009) Systemic manifestations and comorbidities of COPD. Eur Respir J 33: 1165-85.

2. Decramer M, Rennard S, Troosters T, Mapel DW, Giardino N, et al. (2008) COPD as a lung disease with systemic consequences clinical impact, mechanisms, and potential for early intervention. COPD 5: 235-56.

3. Eickhoff P, Valipour A, Kiss D, Schreder M, Cekici L, et al. (2008) Determinants of systemic vascular function in patients with stable chronic obstructive pulmonary disease. Am J Respir Crit Care Med 178: 1211-8.

4. Brekke PH, Omland T, Holmedal SH, Smith P, Søyseth V (2008) Troponin T elevation and long-term mortality after chronic obstructive pulmonary disease exacerbation. Eur Respir J 31: 563-70.

5. Vahanian A, Baumgartner H, Bax J, Butchart E, Dion R, et al. (2007) Guidelines on the management of valvular heart disease. Eur Heart J 28: 230-68.

6. Burger CD (2008) Pulmonary hypertension in COPD: a review and consideration of the role of arterial vasodilators. COPD 6: 137-44.

7. Chaouat A, Naeije R, Weitzenblum E (2008) Pulmonary hypertension in COPD. Eur Respir J 32: 1371-85.

8. Solidoro P, Boffini M, Lacedonia D, Scichilone N, Paciocco G, et al. (2014) Pulmonary hypertension in COPD and lung transplantation: timing and procedures. Minerva Med 105: 1-7.

9. Cuttica MJ, Kalhan R, Shlobin A, Ahmad S, Gladwin M, et al. (2010) Categorization and impact of pulmonary hypertension in patients with advanced COPD. Respir Med 104: 1877-82.

10. Hanaoka M, Ideura G, Ito M, Urushihata K, Koizumi T, et al. (2008) Pulmonary haemodynamic changes in patients with severe COPD. Respirology 13: 919-22.

11. Naeije R, Boerrigter BG (2013) Pulmonary hypertension at exercise in COPD: does it matter? Eur Respir J 41: $1002-4$.

12. Lewis GD, Bossone E, Naeije R, Grünig E, Saggar R, et al. (2013) Pulmonary vascular hemodynamic response to exercise in cardiopulmonary diseases. Circulation 128: 1470-9.

13. Chen W, Hong YQ, Meng ZL (2014) Bioinformatics analysis of molecular mechanisms of chronic obstructive pulmonary disease. Eur Rev Med Pharmacol 18: 3557-63.

14. Jin Q, Chen Y, Lou Y, He X (2013) Low Serum retinol-binding protein-4 levels in acute exacerbations of chronic obstructive pulmonary disease at intensive care unit admission is a predictor of mortality in elderly patients. J Inflamm 10: 31.

15. Gurkan S, Cabinian A, Lopez V, Bhaumik M, Chang JM, et al. (2013) Inhibition of type I interferon signalling prevents TLR ligand-mediated proteinuria. J Pathol 231: 248-56.

16. Moghimpour BF, Vallejo JG, Rezaei N (2012) Toll-like receptor signaling pathways in cardiovascular diseases: challenges and opportunities. Int Rev Immunol 31: 379-95.

17. Huang H, Lee SH, Ye C, Lima IS, Oh BC, et al. (2013) ROCK1 in AgRP neurons regulates energy expenditure and locomotor activity in malemice. Endocrinology 154: 3660-70.

18. Zhou X, Wei M, Wang W (2014) MicroRNA-340 suppresses osteosarcoma tumor growth and metastasis by directly targeting ROCK1. Biochem Biophys Res Commun 437: 653-8.

19. Hickam JB, Cargill WH (1947) Pulmonary arterial pressures in congestive failure and emphysema. Am J Med 3: 504.

20. Tolle JJ, Waxman AB, Van Horn TL, Pappagianopoulos PP, Systrom DM et al. (2008) Exercise-induced pulmonary arterial hypertension. Circulation 118: 21839 .

21. Hawrylkiewicz I, Izdebska-Makosa Z, Grebska E, Zielinski J (1982) Pulmonary haemodynamics at rest and on exercise in patients with idiopathic pulmonary fibrosis. Bull Eur Physiopathol Respir 18: 403-10.

22. Holverda S, Rietema H, Westerhof N, Marcus JT, Gan CT, et al. (2009) Stroke volume increase to exercise in chronic obstructive pulmonary disease is limited by increased pulmonary artery pressure. Heart 95: 137-41.

23. Global Initiative for Chronic Obstructive Lung Disease (2017) Global initiative for chronic obstructive lung disease pocket guide to COPD diagnosis, management, and prevention a guide for health care professionals, 2017 report, USA.

24. Bruce RA (1971) Exercise testing of patients with coronary heart disease. Principles and normal standards for evaluation. Ann Clin Res 3: 323-32.

25. Hopkins N, Mc Loughlin P (2002) The structural basis of pulmonary hypertension in chronic lung disease: remodelling, rarefaction or angiogenesis? J Anat 201: 335-48.

26. Machado RF, Mack AK, Martyr S, Barnett C, Macarthur P, et al. (2007) Severity of pulmonary hypertension during vaso-occlusive pain crisis and exercise in patients with sickle cell disease. Br J Haematol 136: 319-25.

27. Varga J, Palinkas A, Lajko I, Horváth I, Boda K, et al. (2016) Pulmonary Arterial Pressure Response During Exercise in COPD: A Correlation with C-Reactive Protein [hsCRP]. Open Respir Med J 10: 1-11.

28. Kovacs G, Berghold A, Scheidl S, Olschewski H (2009) Pulmonary arterial pressure during rest and exercise in healthy subjects: a systematic review. Eur Respir J 34: 888-94. 
29. Lam CS, Borlaug BA, Kane GC, Enders FT, Rodeheffer RJ, et al. (2009) Age-associated increases in pulmonary artery systolic pressure in the general population. Circulation 119: 2663-70.

30. Spiekerkoetter E, Fabel H, Hoeper MM (2002) Effects of inhaled salbutamol in primary pulmonary hypertension. Eur Respir J 20: 524-8.

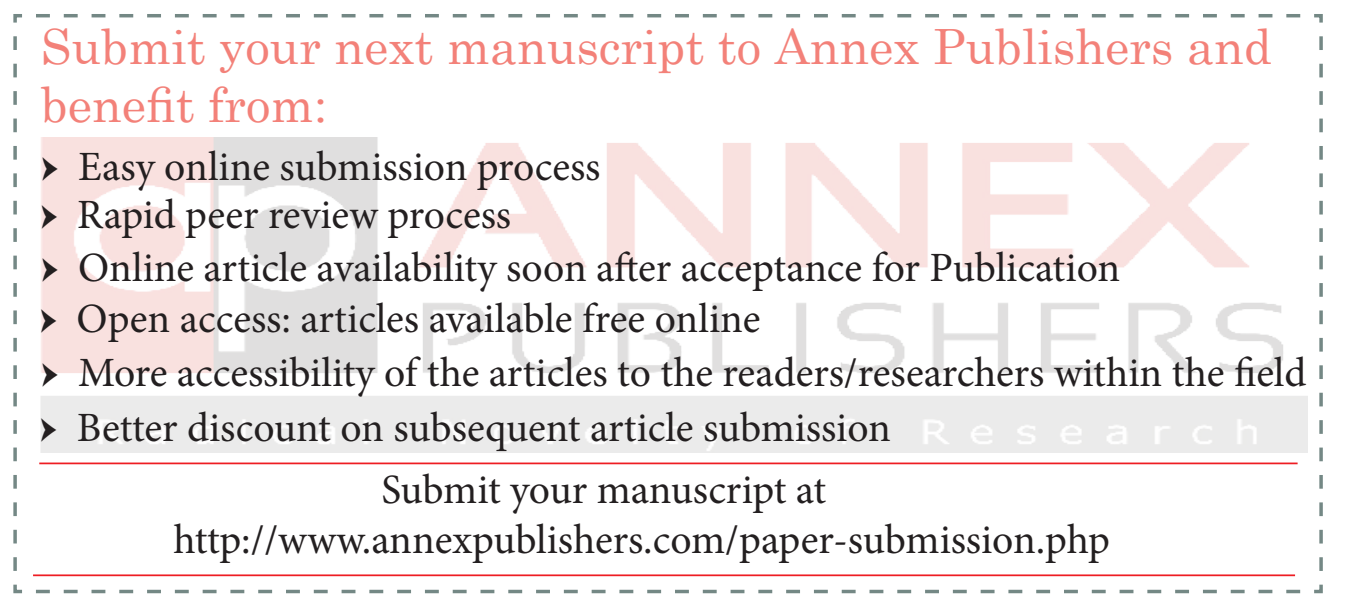

